\title{
HOW TO SEARCH FOR CHEMICAL BIOSIGNATURES ON OCEAN WORLDS
}

A White Paper for the 2020 Decadal Survey on Planetary Science and Astrobiology

Peter Willis ${ }^{1 *}$, M. Fernanda Mora ${ }^{1}$, Aaron Noell ${ }^{1}$, Jessica Creamer $^{1}$, Florian Kehl ${ }^{1}$, Konstantin Zamuruyev ${ }^{1}$, Elizabeth Jaramillo ${ }^{1}$, Miranda Kok ${ }^{1}$, Zuzana Cieslarova ${ }^{1}$, Mauro Sergio Ferreira Santos ${ }^{1}$, Nathan Oborny ${ }^{1}$, Tomas Drevinskas ${ }^{1}$, Mircea Badescu ${ }^{1}$, Jurij Simcic ${ }^{1}$, Sarah Waller $^{1}$, Stojan Madzunkov ${ }^{1}$, Christian Lindensmith ${ }^{1}$, Will Brinckerhoff ${ }^{2}$, Tori Hoehler ${ }^{3}$, Richard Quinn ${ }^{3}$, Antonio Ricco ${ }^{3}$, Sue Lunte ${ }^{4}$, and Alexis Templeton ${ }^{5}$

1 Jet Propulsion Laboratory, California Institute of Technology, Pasadena CA ${ }^{2}$ NASA Goddard Space Flight Center, Greenbelt MD

${ }^{3}$ NASA Ames Research Center, Moffett Field CA

${ }^{4}$ University of Kansas, Lawrence KS

${ }^{5}$ University of Colorado, Boulder CO

*peter.a.willis@jpl.nasa.gov, tel: (818)-354-1297 


\section{Executive Summary}

Chemistry measurements are essential in the search for life. Although precisely where and when to make these measurements is the subject of a number of other white papers and mission studies in this decadal survey, there is a strong community agreement upon why these measurements are critical in the search for life on ocean worlds in the coming decade. Even what to search for, at least at a high level, enjoys broad agreement: biosignatures can come in the form of complexity in single molecules, or they can be expressed in the relative abundances of organic molecules present in a sample. The remaining seemingly open question is, do we know how to conduct this search? The focus of this white paper is to clearly explain that the answer to this question is a resounding yes. We know precisely how to search for chemical biosignatures, including those very different from the ones found on Earth, during spaceflight missions to ocean worlds like Europa or Enceladus. Critically, we also know how to maximize the sensitivity of a chemical analysis system to detect biosignatures, and how to maximize the number of different biosignatures that could be recognized by it. To do so requires sample ingestion, extraction, liquid sample handling, separation science, and finally, detection. Separation science is critical in order to tease apart complex organic chemistry and by using multiple detection modes, a wide range of compound classes can be targeted. One essential detection mode to implement following separation is mass spectrometry (MS) in order to detect a variety of organics and to characterize potential unknowns. Additionally, the liquid sample handling should also be capable of routing sample to other types of analysis, including inorganic chemical analysis to provide sample context information. For extant life detection, these chemistry measurements are most powerfully complemented by the direct search for cellular life forms using microscopy. With these combined measurements, we maximize our chances of acquiring the extraordinary evidence required to establish the presence of life beyond Earth. Conveniently, physical integration of all of these measurements (chemistry, cellularity, and context) on ocean worlds can be achieved using microfluidic technologies. The optimization of instrument suites of this kind, for the study of relevant ocean world analog samples, is a key area of necessary development in order to be ready for astrobiology missions in the coming decade.

\section{The Fundamental Role of Chemical Analysis in the Search for Life}

Chemistry measurements of organic and inorganic matter are indispensable in the search for life beyond Earth.

The scientific community has recently engaged in numerous studies, workshops, and conferences to explore how to best search for life on ocean worlds. Two things that all of studies agree upon are: (1) any successful life detection strategy will rely on multiple lines of evidence, such as those described in the Ladder of Life Detection, ${ }^{1}$ and (2) highly sensitive and specific chemical analysis is an essential component of all searches.

To be clear, both of these points were appreciated even during the Viking missions to Mars. Yet although Viking included a gas chromatography mass spectrometer (GC-MS) instrument for organic molecule detection, due to an insufficient understanding of sample context, detected organics were incorrectly attributed to terrestrial contamination. ${ }^{2} \mathrm{~A}$ lack of knowledge of sample context also led to ambiguous results from the Viking biology experiments, which ultimately did not provide compelling evidence for life. It was not until 
three decades later, during the Phoenix mission (which was specifically aimed at chemical characterization of the Martian regolith) that this crucial chemical context was provided, and the results of the Viking experiments were finally understood. ${ }^{3}$ This experience underscores not only the need for organic analysis in life detection, but also the need for complementary inorganic chemistry measurements to place organic analysis in context.

Hence, it is vitally important that the next generation of life detection missions to ocean worlds builds upon a critical lesson in how to search for chemical biosignatures learned on Mars: chemical analysis is crucial not only for the detection of biosignatures, but also for placing experimental results in the context of their environment. Inorganic chemistry measurements such as total conductivity, sample $\mathrm{pH}$, the identities and concentrations of soluble ions (salts), electron donors and acceptors (redox-active species), and reactive species can be used to characterize chemical sources of energy available in the system. This information can also be used to assess whether the detected organic molecules (or their distributions) have been altered by the environment (thus potentially masking their initial biological fingerprint). Additionally, these measurements are also invaluable in identifying potential measurement interferences during downstream organic analysis.

While the inorganic measurements are critical to enabling mission success and contextualizing organic molecules identified, it is the organic measurements that could provide the most direct and powerful evidence for life. So let's briefly review what we should look for in an organic chemistry-focused search for life on ocean worlds, as this directly informs how we will perform our search. To begin, we consider that the chemical logic employed by life on Earth is universal. That is, the chemical properties of carbon (wherever present in the universe) make it uniquely well suited as a scaffolding molecule, providing strength, flexibility, and diversity to carbon-containing organic compounds that is not replicated by other atoms. ${ }^{4}$ We also know that abiotic carbon-based organic chemistry exists throughout the universe, and therefore, finding organic compounds on another world is not the same thing as finding life. We must focus on a deeper level of characterization that considers how the presence of life impacts the composition, structure, and diversity of organic molecules present, and how those impacts can be identified as biosignatures.

The identification and description of biosignatures is fundamental to the chemical approach of life detection and can be summarized or explained in many equivalent ways, but fundamentally these descriptions amount to three types of biosignatures: ${ }^{1,6}$

A) Large single molecules: life generates large complex molecules whereas abiotic processes favor smaller and simpler ones,

B) Distributions of smaller molecules: life produces patterns in collections of relatively simple organic molecules whereas abiotic processes produce random distributions, and

C) Skewed isotopic ratios of atoms within molecules: life actively sequesters molecules from its environment, skewing the isotopic ratio of atoms from those produced through abiotic equilibria.

The most compelling evidence for life comes from the identification of a combination of these biosignatures. The least ambiguous individual line of evidence would come from (A) the discovery of large, complex molecules for which there is no potential abiotic mechanism for formation. The discovery of such molecules would not only indicate the presence of biotic synthesis, but may also provide insights into a novel system of biochemistry. To make this search truly universal, instrumentation should be capable of 
identifying both complex terrestrial biotic compounds as well as complex molecules not witnessed in terrestrial biology. Similarly compelling is (B), the identification of ordered distributions of organic molecules. ${ }^{5}$ For this biosignature, it is not simply enough to detect molecules, it is also necessary to quantify the relative abundance of organic molecules present. (Note the measurement of the chiral ratios of amino acids is an example of this type of biosignature.) The search for order in collections of organic molecules has the added benefit that in the absence of life, there is significant scientific value in understanding the role of organic chemistry on other worlds. Finally, the use of bulk isotopic measurements $(C)$ of element types present in a sample is the most ambiguous form of evidence of the three. This ambiguity can be reduced considerably by performing compound-specific isotopic measurements to search for evidence regarding the source of precursors and their mechanisms of assembly. However, to use these measurements to provide direct evidence of life requires considerable information about the massbalance in the system to be interrogated, which is beyond the mission capabilities typically envisioned in the next decade. Hence, in the remainder of this paper, as was done in the Europa Lander Mission SDT report, ${ }^{6}$ we will focus our discussion on the first two classes of biosignatures. The following sections of this paper address the necessary considerations for integrating these analyses into a single analytical payload for astrobiology missions to ocean worlds in the coming decade.

\section{The Inescapable Need for Sample Handling}

To address the big questions in astrobiology during future in situ missions to ocean worlds, and to search for life, instrumentation must ingest samples prior to analysis.

Mission designs to search for chemical biosignatures in the coming decade would be drastically simplified if we possessed technologies akin to the tricorder envisioned in the fictional voyages of Star Trek. The tricorder is portrayed as a hand-held unit capable of determining the complete chemical composition of an object in a non-invasive fashion the ultimate non-sampling instrument. Unfortunately, in reality, state-of-the-art nonsampling instrumentation is not so capable, especially for analysis of trace levels of organics. Although many remote-analysis optical and spectroscopic techniques can provide essential context measurements, these techniques lack the analytical sensitivity and the specificity required for life detection in environments with extremely low concentrations of organics. This was also highlighted in the Mars 2020 Science Definition Team (SDT) report ${ }^{7}$ (Figure 1) and in the Europa Lander SDT report. ${ }^{6}$

Figure 1 (adapted from the Mars 2020 mission SDT report ${ }^{7}$ ) provides an overview of different techniques that could be used during spaceflight missions in the search for organic biosignatures. As shown, there is a correlation between sensitivity (estimated detection limits), ability to characterize organics (indicated by colors), and the amount of sample processing (low to high) that needs to be performed in order to acquire this data. Sample handling is necessary in order to obtain sufficient sensitivity and high characterization power in the search for chemical biosignatures. Higher sensitivity can be obtained because sample handling affords the possibility of teasing apart the organic compounds of interest from other materials present in the sample, collectively known as the sample matrix. These other materials are known to interfere with analytical instrumentation in a host of ways (matrix effects) that reduce the sensitivity and can lead 
to ambiguity in interpretation of results (reinforcing again the need for simultaneous inorganic analysis).

Furthermore, for missions to ocean worlds, the sample-handling system must be capable of processing liquids. Liquid sample handling is required to access the molecules we need to characterize. Additionally, using liquid sample handling, samples can be prepared and shared for a wide variety of analyses (including a microscopic survey for cellular materials), and can be readily interfaced to gas-phase analysis systems. This could potentially give incredibly powerful evidence of life by examining the same sample using both a chemical analyzer and a microscopy instrument.

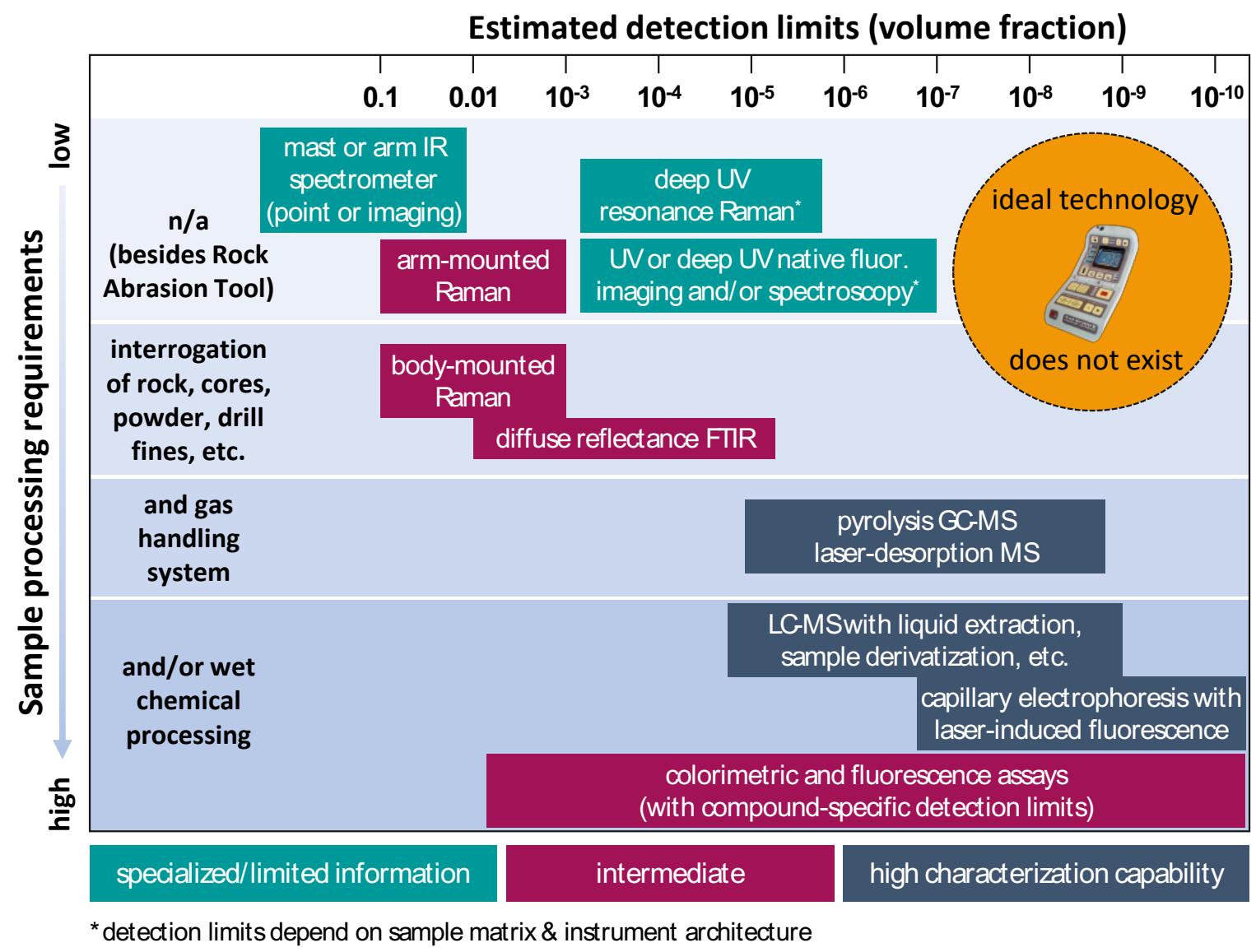

Figure 1. Examples of techniques that can be used in the search for organic biosignatures. There is a correlation between detection limits for organics, characterization capability, and the amount of sample processing that is required. For reference, the Europa Lander SDT report ${ }^{6}$ sets the limit of detection for organics at volume fraction levels of 10-9. Figure reproduced from Mars 2020 SDT Report. ${ }^{7}$ (fluor, fluorescence; FTIR, Fourier transform IR; GC, gas chromatography; LC, liquid chromatography; MS, mass spectrometry.)

\section{The Need for Separation Science in Multiple States of Matter}

To maximize our chances of identifying molecular biosignatures during potential future ocean worlds missions, we need to effectively implement separation science.

The primary benefit of sample handling instruments in the search for life is their ability to tease the sample apart and focus on the biosignature molecules of interest. The most 
powerful way to accomplish this is through separation science. Separation science can be used to examine the individual components of complex mixtures, and focuses on the development of methods that enable the sensitive and simultaneous detection of species with a wide range of molecular masses, functional groups and levels of molecular organization. Separation processes can be applied in the gas, liquid, or supercritical phases, and can be based upon a variety of physical phenomena.

Previous missions have successfully implemented gas-phase separation science (GC) with MS detection for the analysis of organic compounds with significant vapor pressure (compounds that can be transferred into the gas phase by heating, without being destroyed in the process). This type of GC-MS analysis by the SAM instrument suite, for example, was used to detect and identify thiophene on Mars. ${ }^{8}$ However, it must be noted that volatile organic compounds represent only part of the biomass associated with life. The majority of the compounds of terrestrial life are water-soluble and have low vapor pressure (Figure 2). Even if life is chemically very different from ours on ocean worlds, we can still confidently expect that water-soluble compounds would comprise essential building blocks in the molecular machinery of life. Water-soluble, low-volatility compounds cannot be analyzed in their natural form using gaseous techniques (Figure 2). The obvious solution to this issue is to perform separation science directly in the liquid phase. Gas-phase measurements are still critical, as volatile species could provide important information about life, but they can be implemented in a parallel path from a centralized sample handling system. By using a combination of gas- and liquid-based separation science, an extremely wide range of organic and inorganic compounds can be characterized (Figure 2).

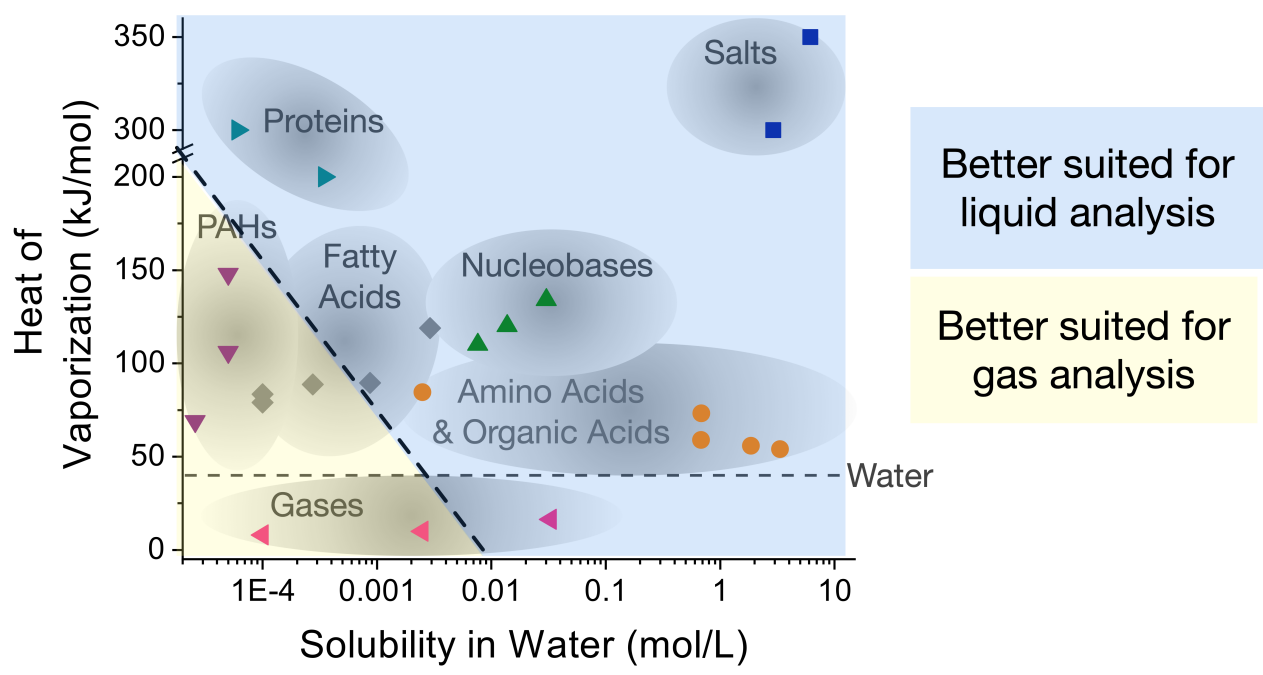

Figure 2. "Molecular Space" relevant in the search for life. The goal of chemical analysis is to provide the maximum coverage of this entire space at the highest possible sensitivity. Different analytical techniques utilizing separation science coupled to multiple detectors can be applied to different regions of this space.

\section{Optimizing the Detection of Separated Chemical Species: Casting the Widest and Tightest Possible Nets in "Molecular Space"}

In order to survey the widest possible range of organic molecules and biosignatures during missions, we must select and optimize detection methods based on the target molecules and sample type. 
The next step in the process of chemical analysis, after a mixture has been separated into its components, is to detect whatever molecules are present. In order to conduct a well-rounded life detection mission, it is necessary to survey the widest possible range of molecules at the highest level of sensitivity possible. An approach that meets both of these requirements would ensure that the majority of organic molecules in an environment could be detected, even if they exist at low concentrations. This approach is analogous to fishing with a wide but tightly woven net. The wide net (a diverse and complementary set of detection methods) improves the chance of catching fish (biosignatures), while the tightly woven net (high sensitivity technique) ensures that even the faintest biosignature does not go undetected.

Mass spectrometry is a uniquely powerful detection method due to its ability to detect a wide range of organic molecules associated with biosignatures, both as complex molecules and as molecular distributions. However, to analyze a sample with MS, molecules must first be liberated from the sample, and ionized in the MS. These two steps - extraction and ionization - vary significantly in their efficiency depending on the type of sample, the ionization method, and the molecular properties of the target molecule. In short, the MS - while exquisitely powerful - is not a universal chemical detection system.

One example where an alternative form of detection is preferred over MS detection is the chiral analysis of amino acids. In this case, the use of laser-induced fluorescence (LIF) detection, which is two orders of magnitude more sensitive than MS for amino acid analysis $^{9}$ is a particularly appealing choice. Similarly, MS is not well suited for the detection of inorganic salts or metals, which as we established, are critical to measure with the same analysis suite. Instead, contactless conductivity detection, performed upstream of MS analysis, can be used to identify these inorganic compounds. ${ }^{10}$ Here we have highlighted three complementary detection methods (MS, LIF, and conductivity) to provide a well-rounded instrument suite for life detection, but there are additional methods that may prove suitable, depending on the overall design of the instrument suite. Nonetheless, the considerations discussed here still apply - a wide range of molecules need to be detected with a high level of sensitivity.

\section{Developing Chemistry Laboratories for Spacecraft}

The scientific needs of an astrobiology mission can be used to produce a generic "blueprint" for a chemistry-focused analytical payload.

With the fundamental requirements on how to search for chemical biosignatures established, we can now lay out what a life detection suite for exploring ocean worlds should look like (Figure 3 ). Such a suite should:

1) receive solid or liquid samples and perform liquid/gas extractions,

2) perform liquid sample handling on samples and extracts,

3) determine the inorganic composition of the sample in the liquid phase,

4) perform separations (liquid and gas) coupled to mass spectrometry detection, and

5) perform separations (liquid) coupled to conductivity and fluorescence detection.

To do all of these we will have to incorporate liquid plumbing as an intrinsic part of spacecraft sample handling and science payloads. By using fluid distribution systems, these liquids could be routed to a wide range of complementary instrumentation to meet the science goals and environmental constraints of a specific mission. By planning 
spacecraft mission operations around the general-purpose architecture described here, it is possible to begin intra-instrument integration (and cross-calibration) very early in the process, mitigating integration risks at later phases of the project. We strongly advise this form of "instrument suite" integration and testing using standardized samples: after all, this is how investigations will be executed during missions. These recommendations are also echoed in the community White Paper from the Network for Life Detection (NfoLD).

Previous flight instruments and ongoing technology maturation efforts have positioned the community to incorporate these and related technologies into a liquid handling payload for life detection missions envisioned in the 2020 Planetary Decadal Survey. Notably, all of the technologies identified in Figure 3 have ongoing development efforts funded by high TRL (TRL 4+) programs like MatISSE, ICEE-2, and COLD-Tech.

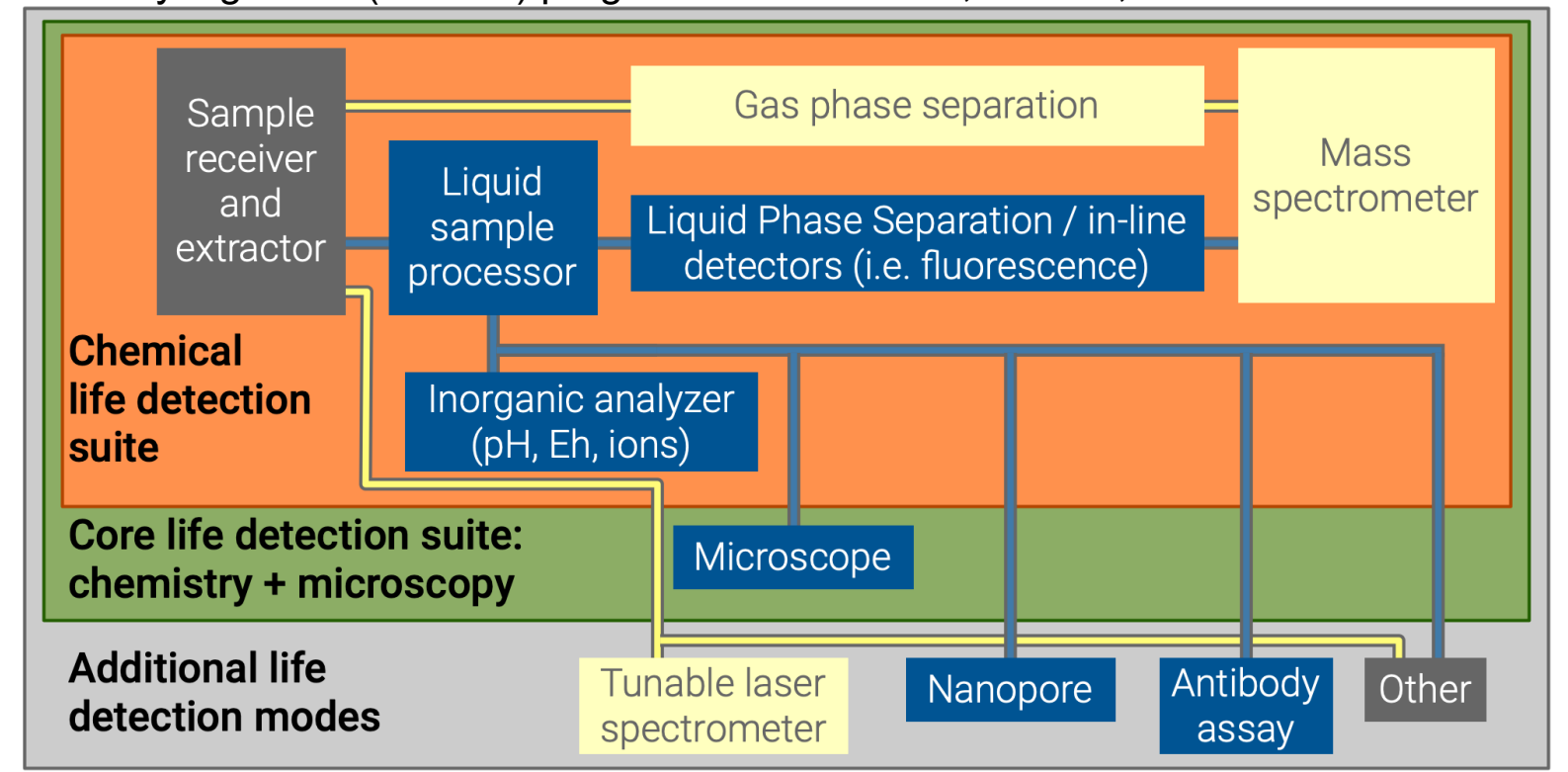

Figure 3. Blueprint for the life detection instrument suite presented here. The system architecture is driven by the liquid-based processes that it must perform on a sample. We must build and test this architecture on natural samples, in order to be ready to interpret data acquired during an ocean worlds mission.

In addition to the chemical characterization approaches mentioned above, it is important to reemphasize that the most striking sign of life on another world could come from evidence of cellular activity. This type of investigation is described in detail in a related White Paper by Lindensmith et al. Taken jointly, detection of organic biosignatures, inorganic chemical characterization, and visualization of cells could provide the extraordinary evidence necessary to substantiate the claim that life exists beyond Earth.

\section{References:}

[1] Neveu, M. et al. Astrobiology, 2018. [2] Guzman, M. et al. J. Geophys. Res. Planets, 2018. [3] Hecht, M. H. et al. Science, 2009. [4] Hoehler, T. et al. Planetary Astrobiology 2020. [5] Lovelock, J. E. Nature, 1965. [6] Hand, K. P. et al. Report of the Europa Lander SDT; NASA, 2017. [7] Mustard, J. F. Mars 2020 Mission Science Definition Team Report; NASA, 2013. [8] Eigenbrode, J. et al. Science, 2018. [9] Creamer, J. S. et al. Anal. Chem., 2017. [10] Ferreira Santos, M. S. et al., Electrophoresis, 2018.

Acknowledgements: The research was carried out at the Jet Propulsion Laboratory, California Institute of Technology, under a contract with the National Aeronautics and Space Administration (80NM0018D0004). 